\title{
Screening for metabolic syndrome in long-term psychiatric illness: Audit of patients receiving depot antipsychotic medication at a psychiatry clinic
}

\author{
Carminda O'Callaghan \\ Aaron Y.L. Liew \\ Mohd Shazli Draman Yusof \\ Richard Duffy \\ Eugene G. Breen \\ Brendan Kinsley \\ Brendan D. Kelly \\ Department of Adult Psychiatry, \\ University College Dublin \\ IRELAND
}

\begin{abstract}
Background and Objectives: Metabolic syndrome (visceral obesity, dyslipidaemia, hyperglycaemia, hypertension) is a substantial public health problem, especially amongst individuals receiving antipsychotic medication.

Methods: We studied routine screening practices for metabolic syndrome amongst psychiatry outpatients receiving injected depot anti-psychotic medication at a clinic in Dublin, Ireland.

Results: Our initial audit $(\mathrm{n}=64)$ demonstrated variable levels of documentation of criteria for metabolic syndrome in outpatient files; e.g. weight was recorded in $1.6 \%$ of files, serum high density lipoprotein in $12.5 \%$. As our intervention, we introduced a screening check-list comprising risk factors and criteria for metabolic syndrome, based on the definition of the International Diabetes Federation. Re-audit $(n=54)$ demonstrated significantly improved levels of documentation; e.g. weight was recorded in $61.1 \%$ of files. Notwithstanding these improvements, only $11(20.4 \%)$ of 54 patient files examined in the re-audit, contained sufficient information to determine whether or not the patient fulfilled criteria for metabolic syndrome; of these, 3 patients $(27.3 \%)$ fulfilled criteria for metabolic syndrome. There was, however, significant additional morbidity in relation to individual criteria (waist circumference, serum triglyceride level, systolic blood pressure and serum fasting glucose).

Conclusions: We recommend enhanced attention be paid to metabolic morbidity in this patient group.
\end{abstract}

Received: 30 July 2010

Revised: 11 February 2011

Accepted: 1 April 2011 


\section{Introduction}

Metabolic syndrome comprises visceral obesity, dyslipidemia, hyperglycaemia and hypertension ${ }^{1}$ (Table 1). Metabolic syndrome is one of the world's largest public health problems, with an estimated prevalence of $20 \%-25 \%$ in the general population ${ }^{2-4}$. It is associated with a three-fold increase in risk of myocardial infarction or cerebro-vascular accident, and two-fold increase in risk of dying from either ${ }^{3,4}$.
Metabolic syndrome is especially prevalent amongst individuals with mental illness $^{2}$. In the first instance, individuals with mental illness are significantly more likely to smoke cigarettes compared to the general population ${ }^{5}$ and this increases risk of metabolic syndrome ${ }^{6}$. Individuals with mental illness are less likely to attend to their physical health, further increasing risk ${ }^{7}$. In addition, individuals receiving neuroleptic medications are at even greater risk of metabolic syndrome compared to individuals receiving no psychotropic medication (odds ratio

Table 1

The International Diabetes Federation (IDF) criteria for metabolic syndrome ${ }^{3}$

Criterion Value

\begin{tabular}{lll}
\hline A & Central obesity & $\begin{array}{l}\text { Body mass index (BMI) greater than 30 kilograms/metres } \\
\text { or waist circumference greater than or equal to } 94 \\
\text { centimetres in males and } 80 \text { centimetres in females }\end{array}$ \\
\hline B & Serum triglyceride level & Greater than or equal to 1.7 milimoles per litre \\
\hline C & Serum high density lipoprotein & $\begin{array}{l}\text { Less than } 1.03 \text { milimoles per litre in males, or less than } \\
1.29 \text { milimoles per litre in females }\end{array}$ \\
\hline D & Systolic blood pressure & $\begin{array}{l}\text { Greater than or equal to } 130 \text { millimetres of mercury } \\
\text { or diastolic blood pressure greater than or equal to } \\
\end{array}$ \\
& Fasting glucose & Greater than or equal to 5.6 milimoles per litre.
\end{tabular}

Notes: Adapted from the International Diabetes Federation criteria for metabolic syndrome ${ }^{3}$.

In order to have a diagnosis of metabolic syndrome, the patient must fulfil criterion A and two of the other four criteria (B-E).

$3.7,95 \%$ confidence interval $1.7-7.9, \mathrm{p}=$ $0.001)^{8}$. Overall, the prevalence of metabolic syndrome in schizophrenia is estimated as between $30 \%$ to $40 \%$, although some suggest a prevalence as high as $63 \%{ }^{7}$.

An up-to-date overview of scientific and clinical aspects of the metabolic syndrome is provided by Byrne ${ }^{9}$; a detailed review of the relationship between metabolic syndrome and psychiatric illness by Mendelson ${ }^{10}$; and a focussed examination of metabolic effects of psychotropic drugs by Thakore and Leonard $^{11}$. Much recent literature relating to metabolic syndrome has focussed on second generation oral antipsychotics, such as clozapine $^{12,13}$.

We performed a quality improvement project focussing on screening for metabolic syndrome amongst individuals receiving injected receiving depot antipsychotic medication at a general adult psychiatry outpatient clinic. 


\section{Methods}

This study was a quality improvement project based at the Mater Misericordiae University Hospital Psychiatry Outpatient Clinic. This clinic provides psychiatric services to all adults residing in a geographically-defined inner-city catchment area; this catchment area is the most socio-economically disadvantaged in Ireland, with over $50 \%$ of its population in the most deprived socio-economic category ${ }^{14,15}$.

The inclusion criteria were that the patient was (1) attending the clinic; (2) aged 18 years or over; (3) receiving injected depot anti-psychotic medication; and (4) willing to participate. Exclusion criteria were that the patient was (1) aged under 18 years; and (2) unwilling to participate. The patient could be receiving other medication in addition to injected depot anti-psychotic medication (e.g. oral antipsychotic medication); this ensured we included all patients receiving injected depot anti-psychotic medication, with the aim of redressing the literature's focus on second generation antipsychotics (such as clozapine), rather than injected depot antipsychotic medication $^{12,13}$.

All patients fulfilling inclusion criteria (n $=64$ ) were invited to participate and audit data were acquired for all; re-audit data were acquired for 54 (eight were no longer attending the clinic and two declined to participate in re-audit).

As this was a quality improvement project, verbal consent was obtained from each patient. This study was undertaken with respect for the right to privacy and in a manner consistent with the guidelines of the World Health Organization and Declaration of Helsinki.

The audit commenced on 1 March 2009; the intervention was performed over a three month period commencing on 1 April 2009; and re-audit was performed on 1 July 2009.

For the audit, the multi-disciplinary team at the clinic devised a single-page checklist for features of, and risk factors for, metabolic syndrome, based on the International Diabetes Federation definition ${ }^{3}$ (Appendix 1). The checklist was created following a series of multi-disciplinary meetings involving all members of the outpatient team including psychiatrists, endocrinologists, trainee doctors, community mental health nurses, psychiatry social worker, occupational therapist and administrative staff.

We commenced the audit by examining clinical files of all patients $(n=64)$ who were receiving injected depot anti-psychotic medication at the clinic on 1 March 2009. We introduced this check-list into clinical practice at the clinic over a three month period. Nursing staff, psychiatrists and trainee doctors were responsible for filling out the checklist. As each patient received their injected depot antipsychotic medication, clinical and anthropometric data were recorded, and a phlebotomy appointment arranged at the local hospital within the following week. After three months, we performed re-audit, in which we sought to review the same 64 patient files.

\section{Results}

Sixty-four patients were included in the audit. Twenty-seven (42\%) were female. Mean age was 54.4 years (range, 21-76; standard deviation [SD] 12.1). Fifty patients (78\%) had schizophrenia; 10 (16\%) bipolar disorder; and $4(6 \%)$ other diagnoses (e.g. schizoaffective disorder). 
Seventeen patients $(27 \%)$ were on fluphenazine decanoate depot; 16 (25\%) zuclopenthixol decanoate; 16 (25\%) flupenthixol decanoate; 9 (14\%) haloperidol decanoate; and $6(9 \%)$ risperidone microspheres. Mean dose of depot anti-psychotic medication was 530.7 milligrams of chlorpromazine per day (SD 554.7).

Thirty-seven patients $(57.8 \%)$ were also on oral anti-psychotic medication (Table 2). Amongst those on oral anti-psychotic medication, mean dose of oral anti-psychotic medication was 567.2 milligrams of chlorpromazine per day (SD 611.6). Total mean dose of anti-psychotic medication (depot and oral) amongst all patients $(\mathrm{n}=64)$ was 858.6 milligrams of chlorpromazine per day (SD 807.2).

Documentation of risk factors for metabolic syndrome varied significantly between risk factors; while $56.2 \%$ of patient files documented alcohol use, only $20.3 \%$ documented family history of premature ischaemic heart disease ( $\mathrm{p}<0.001)$ (Table 3). Regard-

Table 2

Oral anti-psychotic medications in patients receiving depot antipsychotic medication at a general adult psychiatry outpatient clinic (audit and re-audit)

\begin{tabular}{lrrrr} 
Name of oral anti-psychotic medication & \multicolumn{2}{c}{ Audit (n=64) } & \multicolumn{2}{c}{ Re-audit (n=54) } \\
\hline None & $\mathrm{n}$ & $\%$ & $\mathrm{n}$ & $\%$ \\
Olanzapine & 27 & $42.2 \%$ & 22 & $40.7 \%$ \\
Haloperidol & 9 & $14.1 \%$ & 9 & $16.7 \%$ \\
Risperidone & 7 & $10.9 \%$ & 6 & $11.1 \%$ \\
Aripiprazole & 6 & $9.4 \%$ & 6 & $11.1 \%$ \\
Chlorpromazine & 3 & $4.7 \%$ & 3 & $5.6 \%$ \\
Haloperidol and risperidone & 3 & $4.7 \%$ & 2 & $3.7 \%$ \\
Quetiapine & 3 & $4.7 \%$ & 2 & $3.7 \%$ \\
Amisulpiride & 2 & $3.1 \%$ & 1 & $1.9 \%$ \\
Haloperidol and quetiapine & 1 & $1.6 \%$ & 0 & $0 \%$ \\
Risperidone and quetiapine & 1 & $1.6 \%$ & 1 & $1.9 \%$ \\
Sulpiride & 1 & $1.6 \%$ & 1 & $1.9 \%$ \\
\hline
\end{tabular}

ing metabolic syndrome, only $1.6 \%$ of patient files contained patient weight, and the most commonly documented criteria were serum triglyceride level (12.5\%) and serum high-density lipoprotein (12.5\%) (Table 4).

Fifty-four patients were included in the reaudit. Eight were no longer attending and two declined to participate. At re-audit, 21 patients $(39 \%)$ were female. Mean age was 54.6 years (range 21-76; SD 12.2). Forty-three patients
(79\%) had schizophrenia; 9 (17\%) bipolar disorder; and 2 (4\%) other diagnoses.

Fourteen patients $(26 \%)$ were on fluphenazine decanoate depot; 12 (22\%) zuclopenthixol decanoate; 15 (28\%) flupenthixol decanoate; 8 (15\%) haloperidol decanoate; and $5(9 \%)$ risperidone microspheres. Mean dose of depot anti-psychotic medication was 587.3 milligrams of chlorpromazine per day (SD 529.7). 


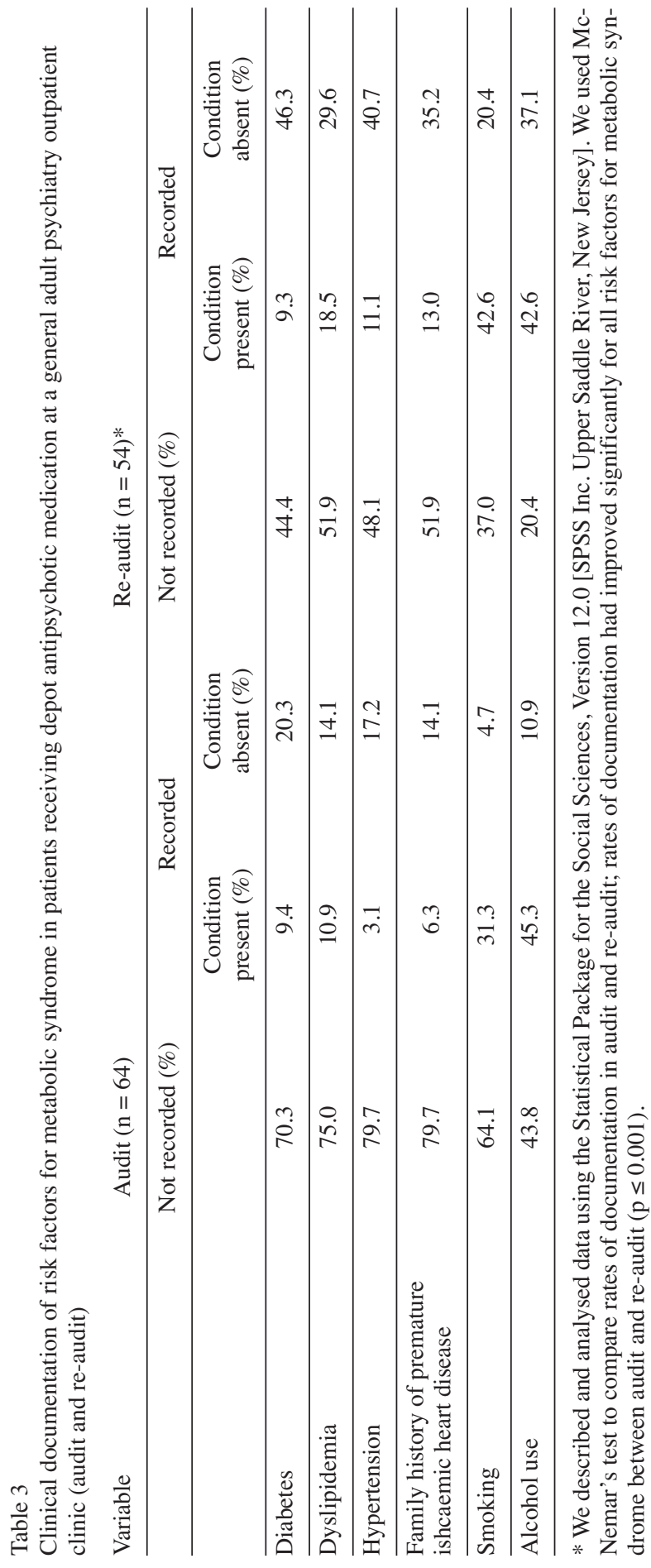




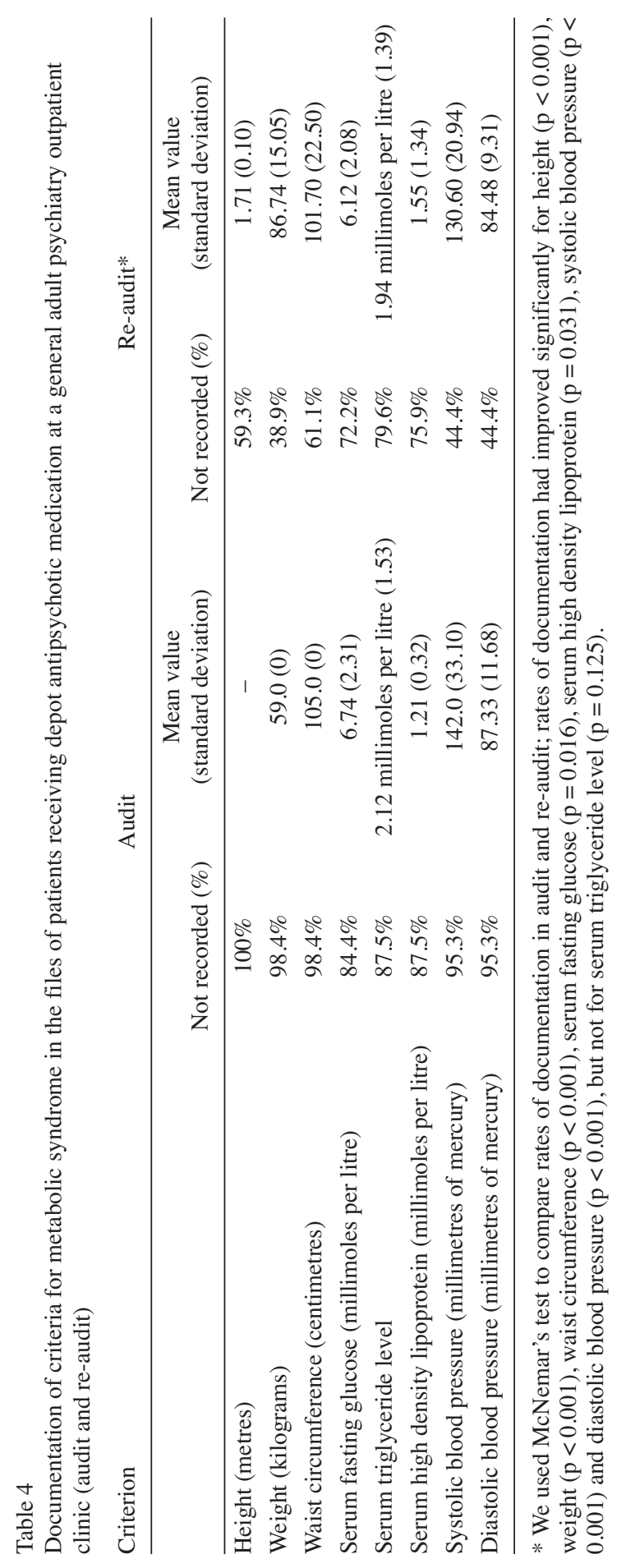


Thirty-two patients $(59.3 \%)$ were also on oral anti-psychotic medication (Table 2). Amongst those who were on oral anti-psychotic medication, mean dose of oral antipsychotic medication was 591.8 milligrams of chlorpromazine per day (SD 595.1). Total mean dose of anti-psychotic medication (depot and oral) was 943.54 milligrams of chlorpromazine per day (SD 780.3).

Documentation of all risk factors for metabolic syndrome had improved significantly, but still varied between risk factors; for example, while $79.6 \%$ had documentation regarding alcohol use, only $48.1 \%$ had documentation regarding family history of premature ischaemic heart disease $(\mathrm{p}<0.001)$ (Table 3).

Documentation of criteria for metabolic syndrome had also improved significantly (Table 4); for example, weight was recorded in approximately $61 \%$ of patient files on reaudit, although serum triglyceride levels were recorded in only approximately $20 \%$. On re-audit, mean BMI was 28.3 kilograms per metre squared (SD 5.6, range 19.940.2); mean serum fasting glucose was 6.12 millimoles per litre; mean serum triglyceride level was 1.94 millimoles per litre; mean serum high-density lipoprotein was 1.55 millimoles per litre; mean systolic blood pressure was 130.6 millimetres of mercury; and mean diastolic blood pressure was 84.5 millimetres of mercury.

Combining information from outpatient files, audit checklists and computerized phlebotomy results at the local hospital, we had information regarding metabolic syndrome criterion A for 22 patients (40.7\%); criterion B for 20 (37.0\%); criterion $\mathrm{C}$ for $21(38.9 \%)$; criterion D for $30(55.6 \%)$; and criterion E for 24 (44.4\%). Overall, we had sufficient information to diagnose or outrule metabolic syndrome for 11 patients (20.4\%) of whom 3 (27.3\%) fulfilled criteria for metabolic syndrome.

\section{Discussion}

Our audit demonstrated variable levels of documentation of criteria for metabolic syndrome in outpatient files and, as our intervention, we introduced a screening checklist comprising risk factors and criteria for metabolic syndrome, based on the definition of the International Diabetes Federation $^{3}$. Re-audit demonstrated significantly improved levels of documentation, although only $20.4 \%$ of patient files contained sufficient information to determine whether or not the patient fulfilled criteria for metabolic syndrome. There was, however, significant morbidity in relation to individual criteria (waist circumference, serum triglyceride level, systolic blood pressure and serum fasting glucose).

This audit addressed an important clinical issue, metabolic syndrome, associated with significant and avoidable mortality, morbidity and health-care costs ${ }^{2,8}$. The audit was performed in the context of a standard general adult psychiatry service, with a focus on the day-to-day practical issues involved in screening for metabolic syndrome (e.g. requesting blood tests, obtaining results, etc.). The mean doses of anti-psychotic medication in both audit and re-audit were within standard prescribing parameters for commonly-used medications (e.g. haloperidol, risperidone, clozapine), thus enhancing generalizability. This audit involved a range of members of the multi-disciplinary team, again reflecting common clinical practice and optimising applicability of findings. Finally, this paper describes a full audit cycle, including audit, intervention and re-audit ${ }^{16,17}$.

This paper has a number of limitations. While the findings from this quality improvement project are not unexpected, and do not modify existing scientific knowledge 
of metabolic syndrome, the project aimed to provide an insight into the realities of quality improvement initiatives in busy, day-to-day clinical practice. The project was, however, very underpowered to look at the prevalence rate of metabolic syndrome, and therefore does not present reliable results in this regard. This project was also dependent on standards of clinical record-keeping; this, however, reflects the reality of clinical practice where the ready availability of the results of a test, rather than whether or not the test was performed, is the key determinant of clinical value derived from the test.

Finally, this audit was based in one psychiatry clinic, in an especially socio-economically deprived inner-city area, and this may limit generalizability to other socioeconomic contexts. Nonetheless, this audit highlights certain issues that appear relevant to most psychiatry outpatient settings, including the necessity for good clinical record-keeping and ready accessibility of test results, as well as the overall importance of screening for metabolic syndrome.

Screening for metabolic syndrome is an especially important issue in this population group: there is strong evidence that individuals with mental illness are less likely to attend to their physical health, compared with individuals without mental illness ${ }^{7}$. Many also smoke ${ }^{5}$ and receive medication associated with various metabolic anomalies and, in some cases, metabolic syndrome ${ }^{11}$. Even if our audit does not support reliable conclusions regarding all criteria for metabolic syndrome, we still found strong evidence of morbidity in relation to specific metabolic parameters; i.e. while there were sufficient data to determine the presence or absence of all criteria in just 11 files, greater numbers had data relating to individual criteria. For example, on re-audit, mean waist circumference (101.7 centimetres) was greater than the threshold required in order to fulfil one of the criteria for metabolic syndrome (for both males and females).

On this basis, we conclude that attendance at the psychiatry clinic presents a unique opportunity for routine health screening amongst this population which is at especially high risk of metabolic morbidity (including diabetes). Moreover, we recommend that special effort be applied to assessment of anthropometric, clinical and biochemical parameters of metabolic syndrome at psychiatry outpatient clinics. Our audit demonstrates clearly the difficulties associated with such extensive screening in day-to-day clinical practice, but the broader literature confirms clearly that the morbidity associated with metabolic syndrome in this group is sufficiently large to justify such greater efforts at detection and management ${ }^{2,7,10}$.

Finally, we recommend that future studies focus not only on factors contributing to metabolic syndrome, such as psychotropic drugs $^{11}$ and smoking 5 , but also the effectiveness of therapeutic measures, especially amongst individuals with mental illness (e.g. exercise, dietary improvement, medication changes, smoking cessation programmes $)^{9}$. Many of these therapeutic measures may be difficult to implement in practice, just as our audit demonstrates that screening measures can be difficult to integrate into day-to-day clinical practice. Nonetheless, the morbidity associated with metabolic syndrome amongst the mentally ill indicates clearly a need for greater basic research, clinical focus and therapeutic intervention in relation to this disorder. 


\section{Appendix 1: Checklist for features of and risk factors for the metabolic syndrome}

Date:

Audit No::

Date of birth:

Gender: Male/Female

Height: centimetres

Weight: kilograms

Psychiatric diagnosis: $\quad$ Schizophrenia

Bipolar affective disorder

Others:

Depot antipsychotic: Name: Nose $\quad$ Frequency

Oral antipsychotic: Name Dose $\quad$ Frequency

Name Dose Frequency

Name Dose $\quad$ Frequency

Risk factors for cardiovascular disease:

Diabetes mellitus

$\mathrm{Y} / \mathrm{N} / \mathrm{ND}$

Dyslipidaemia

$\mathrm{Y} / \mathrm{N} / \mathrm{ND}$

Hypertension

$\mathrm{Y} / \mathrm{N} / \mathrm{ND}$

Family history of premature ischaemic heart disease

$\mathrm{Y} / \mathrm{N} / \mathrm{ND}$

Smoker

$\mathrm{Y} / \mathrm{N} / \mathrm{ND}$

Alcohol intake

$\mathrm{Y} / \mathrm{N} / \mathrm{ND}$

Screening for Metabolic syndrome:

Waist circumference

Serum fasting glucose

Serum triglyceride

Serum high density lipoprotein

Blood pressure

$\begin{array}{ll}\mathrm{Y} / \mathrm{N} / \mathrm{ND} & \text { centimetres } \\ \mathrm{Y} / \mathrm{N} / \mathrm{ND} & \text { millimoles per litre } \\ \mathrm{Y} / \mathrm{N} / \mathrm{ND} & \text { millimoles per litre } \\ \mathrm{Y} / \mathrm{N} / \mathrm{ND} & \text { millimoles per litre } \\ \mathrm{Y} / \mathrm{N} / \mathrm{ND} & \text { millimetres of mercury }\end{array}$

$\mathrm{Y}=$ Yes, $\mathrm{N}=$ No, ND = Not documented. 


\section{Acknowledgements}

We are very grateful to the Editor-InChief and two anonymous peer-reviewers for their comments and suggestions in relation to this paper.

\section{References}

1. Alberti KG, Zimmet P, Shaw J. IDF Epidemiology Task Force Consensus Group. The metabolic syndrome - a new worldwide definition. Lancet 2005; 366(9491): 1059-1062.

2. Thakore J. Metabolic syndrome and schizophrenia. Br J Psychiatry 2005; 186(6): 455-456.

3. International Diabetes Federation. The IDF Consensus Worldwide Definition of the Metabolic Syndrome. Brussels: International Diabetes Federation; 2006.

4. Stern M, Williams K, Golzalez-Villalpando E, Hunt KJ, Haffner SM. Does the metabolic syndrome improve identification of individuals at risk of type 2 diabetes and/ or cardiovascular disease? Diabetes Care 2004; 27(11): 2676-2681.

5. Tanskanen A, Viinamiiki H, Koivumaa-Honkanen H, Jaaskelainen J, Lehtonen J, Smoking among psychiatric patients. Eur J Psychiatry 1997; 11(3): 179-188.

6. Wada T, Urashima M, Fukumoto T. Risk of metabolic syndrome persists twenty years after the cessation of smoking. Intern Med 2007; 46(14): 1079-1082.

7. De Hert M, Schreurs V, Vancampfort D, Van Winkel R. Metabolic syndrome in people with schizophrenia: a review. World Psychiatry 2009; 8(1): 15-22.

8. Mackin P, Bishop D, Watkinson H, Gallagher P, Ferrier N. Metabolic disease and cardiovascular risk in people treated with antipsychotics in the community. Br J Psychiatry 2007 ; 191(1): 23-29.

9. Byrne C. Metabolic Syndrome: Science and Clinical Practice. Oxford: Wiley-Blackwell; 2011.
10. Mendelson SD. Metabolic Syndrome and Psychiatric Illness: Interactions, Pathophysiology, Assessment and Treatment. Burlington, MA: Elsevier; 2008.

11. Thakore J, Leonard BE. Metabolic Effects of Psychotropic Drugs (Modern Trends in Pharmacopsychiatry). Basel: S Karger AG; 2009.

12. Ramaswamy K, Masand PS, Narsrallah HA. Do certain atypical antipsychotics increase the risk of diabetes? A critical review of 17 pharmacoepidemiologic studies. Ann Clin Psychiatry 2006; 18(3): 183-194.

13. Newcomer JW. Second-generation (atypical) antipsychotics and metabolic effects: a comprehensive literature review. CNS Drugs 2005; 19(suppl.1): 1-93.

14. Kelly A, Teljeur C. A new national deprivation index for health and health services research: short report. Small Area Health Research Unit, Department of Public Health and Primary Care. Dublin: Trinity College; 2004.

15. Mental Health Commission. Community mental health services in Ireland: activity and catchment area characteristics. Dublin: Mental Health Commission; 2004.

16. Lelliott, P. Making medical audit effective. Specialty supplement: psychiatry. London: Joint Centre for Education in Medicine/Research Unit of the Royal College of Psychiatrists; 1993.

17. National Health Service, National Institute for Clinical Excellence, Commission for Health Improvement, Royal College of Nursing and University of Leicester. Principles for best practice in clinical audit. Oxon, United Kingdom: Radcliffe Medical Press; 2002.

Author for correspondence:

Carminda O'Callaghan

Department of Adult Psychiatry

University College Dublin

Mater Misericordiae University Hospital

62/63 Eccles Street, Dublin 7

Ireland

Tel. + 35318034474

Fax + 35318309323

E-mail: co_callaghan@hotmail.com 